\title{
Prevalência, distribuição espacial e fatores de risco para cisticercose bovina no estado de São Paulo ${ }^{1}$
}

\author{
Marlon Martins Ferreira², Thais Buranello Revoredo ${ }^{3}$, Juliana Pistore Ragazzi ${ }^{2}$, \\ Vando Edésio Soares ${ }^{4}$, Antônio Sérgio Ferraldo ${ }^{5}$, Rafael Paranhos de Mendonça ${ }^{2}$ \\ e Welber Daniel Zanetti Lopes ${ }^{6 *}$
}

\begin{abstract}
Ferreira M.M., Revoredo T.B., Ragazzi J.P., Soares V.E., Ferraldo A.S., Lopes W.D.Z. \& Mendonça R.P. 2014. [Prevalence, spatial distribution and risk factors for cattle cysticercosis in the state of São Paulo, Brazil.] Prevalência, distribuição espacial e fatores de risco para cisticercose bovina no estado de São Paulo. Pesquisa Veterinária Brasileira 34(12):1181-1185. Curso de Medicina Veterinária, Faculdade Dr. Francisco Maeda, Rodovia Jerônimo Nunes Macêdo Km 1, Ituverava, SP 14500-000, Brazil. E-mail: wdzlopes@hotmail.com

This study aimed to determine the prevalence and geographical distribution as well as the factors and areas of risk associated with bovine cysticercosis in the State of São Paulo. 34.443 cattle, males and females with ages from 18 to 60 months were inspected. The animals were from 97 cities in the state of São Paulo and identified and slaughtered in the period October 2010 to August 2011, in a refrigerator located in Ipuã - SP, under the supervision of SIF 1387. The state of São Paulo was divided into regional centers, and the data of the municipalities belonging to its core, were grouped according to the Department of Agriculture and Food Supply of São Paulo, totaling 13 cores studied. Based on these results, we can conclude that of the 97 cities analyzed, cattle were found positive for the disease in 86. The average prevalence of bovine cysticercosis in the state of São Paulo was $4.80 \%$, while the core inflation Franca and Barretos were the ones with the highest number of cases illness during the analysis period. Moreover, the largest number of cases in these core coincided with the lowest human development index covering education, with the largest acreage of coffee (core Franca) and also as the largest area of cane sugar grown (core Barretos) in these locations, which in turn may indicate that the presence of labor, temporary labor in rural areas , combined with socioeconomic/cultural factors might contribute to the spread and establishment of bovine cysticercosis in these áreas.
\end{abstract}

INDEX TERMS: Cysticercus bovis, bovine cysticercosis, georeferencing, Taenia saginata.

\footnotetext{
${ }^{1}$ Recebido em 18 de setembro de 2014.

Aceito para publicação em 20 de outubro de 2014.

${ }^{2}$ Curso de Medicina Veterinária, Faculdade Dr. Francisco Maeda (Fafram), Rodovia Jerônimo Nunes Macêdo Km 1, Ituverava, SP 14500-000, Brasil.

${ }^{3}$ Médica Veterinária do Serviço de Inspeção Federal (SIF), Rua Moreira Cesar 185, Ipuã, SP 14610-00, Brasil.

${ }^{4}$ Departamento de Medicina Veterinária, Universidade Camilo Castelo Branco (Unicastelo), Av. Hilário da Silva Passo 950, Descalvado, SP 13690970, Brasil.

${ }^{5}$ Departamento de Matemática, Universidade Estadual Paulista (Unesp), Campus de Jaboticabal, Via de acesso Prof. Paulo Donato Castellane s/n, Jaboticabal, SP 14884-900, Brasil.

${ }^{6}$ Departamento de Medicina Veterinária, Universidade Federal de Goiás (UFG), Regional de Jataí, BR-364 Km 192, Parque Industrial, Jataí, GO 75801-615, Brasil.*Autor para correspondência: wdzlopes@hotmail.com
}

RESUMO.- 0 presente trabalho teve como objetivos determinar a prevalência, a distribuição geográfica bem como os fatores e áreas de risco associados à cisticercose bovina no Estado de São Paulo. Foram inspecionados 34.443 animais, machos e fêmeas e com faixas etárias variando entre 18 a 60 meses. Os bovinos eram procedentes de 97 municípios do Estado de São Paulo, devidamente identificados e abatidos no período de outubro de 2010 a agosto de 2011, em um frigorífico localizado na cidade de Ipuã-SP, sob supervisão do SIF 1387. 0 estado de São Paulo foi dividido em núcleos regionais, e os dados dos municípios pertencentes ao respectivo núcleo, foram agrupados, conforme a Secretaria de Abastecimento e Agropecuária de São Paulo, totalizando 13 núcleos estudados. Com base nos resultados encontrados, 
pode-se concluir que dos 97 municípios analisados, foi possível encontrar bovinos positivos para a enfermidade em questão em 86. A prevalência média de cisticercose bovina no estado de São Paulo foi de $4,80 \%$, sendo que os núcleos de Franca e Barretos foram os que tiveram maior número de casos da enfermidade durante o período analisado. Além disso, o maior número de casos nestes núcleos coincidiu com o menor índice de desenvolvimento humano referente à educação, com a maior área de plantio de café (núcleo de Franca) e também como a maior área de cana-de-açúcar cultivada (núcleo de Barretos) nestes locais, o que por sua vez pode indicar que a presença da mão-de-obra temporária no meio rural, aliado a aspectos socioeconômico/cultural, pode estar contribuindo para a disseminação e estabelecimento da cisticercose bovina nestas áreas.

TERMOS DE INDEXAÇÃO: Cysticercus bovis, cisticercose bovina, georeferenciamento, Taenia saginata.

\section{INTRODUÇÃO}

A teníase e a cisticercose são o mesmo parasita, entretanto com denominações diferentes. Para sua disseminação e sobrevivência na natureza, este parasita depende da participação de duas espécies de hospedeiros, a humana e a animal (Wanzala et al. 2002). Sendo assim, Taenia saginata têm como hospedeiro definitivo o homem, que desenvolve no intestino delgado a forma adulta do parasita, enquanto que, a fase de larva deste cestódeo é encontrada nos tecidos de animais, dos quais se destacam os bovinos, seus hospedeiros intermediários (Costa et al. 2012).

Lopes et al. (2011), afirmam que a cisticercose bovina não apresenta elevada importância clínica para a bovinocultura de corte e leite, uma vez que os animais apresentam, geralmente, infecções moderadas e com ausência de sinais. Por outro lado, estes autores enfatizam que, os prejuízos para os produtores ocorrem no abatedouro, resultado do deságio ou mesmo na condenação de carcaças com cisticercos vivos diagnosticados durante a linha de abate. De acordo com Schantz et al. (1994), na América Latina, a perda econômica anual devido a cisticercose bovina é de aproximadamente US\$ 164 milhões, sendo que na África, estão estimadas entre 1000 e 2000 milhões de dólares. Acha \& Szyres (1986) afirmam que esta perda pode ser de US\$ 25/animal infectado nos países em desenvolvimento. No estado do Paraná, entre os anos de 2004-2008, Guimarães-Peixoto et al. (2012) estimaram que foram perdidos em média cerca de $\mathrm{R} \$ 119.626 .428,00$ devido à condenação de carcaças bovina por cisticercose. Além dos prejuízos supracitados, deve-se considerar a importância da carne positiva como fonte de infecção para seres humanos adquirirem a teníase, apesar de ser o homem, o principal responsável por esta enfermidade ocorrer nos animais (Flavigna-Guilherme et al. 2006; Bavia et al. 2012).

Embora as autoridades sanitaristas contribuam para avanços significativos no controle epidemiológico da cisticercose bovina, poucos estudos têm sido conduzidos na tentativa de se estabelecer medidas profiláticas contra esta infecção (Barbosa et al. 2003, Lopes et al. 2014). Por este motivo, o presente trabalho teve como objetivos determi- nar a prevalência, a distribuição geográfica bem como os fatores e áreas de risco associados à cisticercose bovina no Estado de São Paulo, cujos dados foram obtidos de um frigorífico localizado na cidade de Ipuã, sob o controle do Sistema de Inspeção Federal (SIF 1387).

\section{MATERIAL E MÉTODOS}

Foram inspecionados 34.443 bovinos, machos e fêmeas e com faixas etárias variando entre 18 a 60 meses. Os animais eram procedentes de 97 municípios do Estado de São Paulo, devidamente identificados e abatidos no período de outubro de 2010 a agosto de 2011, em um frigorífico localizado na cidade de Ipuã-SP, sob supervisão do SIF 1387.

Os animais foram abatidos conforme tecnologia de produção padrão para bovinos. Os trabalhos de inspeção nas linhas de abate foram feitos por uma equipe composta de agentes de inspeção devidamente treinados para realizar a inspeção post mortem, sob supervisão e responsabilidade do(s) Médico(s) Veterinário(s) do Serviço de Inspeção Federal (SIF). Os exames de rotina desenvolvidos na pesquisa da cisticercose bovina nas linhas de inspeção (cabeça, língua, coração, diafragma e esôfago), foram realizados conforme as normas padronizadas pelo SIF (Brasil 1971, 1980, 1988, 2006).

Caso fossem encontrados cisticercos nos animais nas linhas de inspeção, as lesões eram identificadas e as meias-carcaças, juntamente com as vísceras e cabeça encaminhadas para o Departamento de Inspeção Final (DIF) onde foram examinadas pelo Médico Veterinário responsável, conforme procedimentos regulamentares. Os cistos encontrados foram levados ao laboratório e classificados em duas categorias: vivos e calcificados. Os fragmentos de tecidos ou órgãos contendo os cisticercos foram colhidos e colocados em placas de Petri contendo solução fisiológica. Após serem dissecados cuidadosamente, foram incubados em banho Maria, juntamente com a bile, a $37^{\circ} \mathrm{C}$ por 15 minutos, para promover a evaginação do escólex, por meio da técnica estabelecida por Biond et al. (2000).

0 estado de São Paulo foi dividido em núcleos regionais, e os dados dos municípios pertencentes ao respectivo núcleo, foram agrupados, conforme a Secretaria de Abastecimento e Agropecuária de São Paulo, totalizando 13 núcleos estudados, sendo: Araçatuba, Araraquara, Barretos, Bauru, Campinas, Central, Franca, Marília, Presidente Prudente, Ribeirão Preto, São José do Rio Preto, São José dos Campos e Sorocaba.

Todos os dados coletados foram processados em planilhas do Microsoft Excel, e analisados estatisticamente por meio dos programas de análises estatísticas Epiinfo 3.5.1 e SAEG 9.0. Os dados foram analisados quanto à homogeneidade por meio do teste de Bartlett e normalidade pelo teste de Lilliefors. 0 cálculo da força de associação entre variáveis foi realizado por meio do cálculo da razão de chance de prevalência (Odds ratio-OR).

\section{RESULTADOS}

A avaliação de bovinos 34.443 procedentes de 97 municípios do estado de São Paulo, no período da avaliação permitiu identificar 1.656 animais positivos, representando uma prevalência de $4,8 \%$ para Cysticercus bovis. 0 total de cistos vivos e calcificados encontrados foi $844(45,08 \%)$ e 1028 (54,91\%), respectivamente.

Ao analisar os resultados, por procedência e no período pesquisado, observa-se que dos 97 municípios que tiveram um total de 1387 bovinos abatidos no SIF , somente em 11 deles (Altair, Araraquara, Echaporã, Magda, Narandiba, Nova Castilho, Ribeirão Preto, São João da Boa Vista, Santo Anastacio, Santo Antônio Do Aracanguá e Tabapuã) não foram 
identificados animais positivos para cisticercose, sendo que os 86 restantes que tiveram animais com cisticercose (Fig.1), as prevalências aparentes encontradas nos animais abatidos variaram de 0,73\% em Limeira a 31,58\% em Mendonça.

Os municípios que tiveram maior quantidade de animais para abate, ou seja, que tiveram melhor representatividade amostral foram 15 (Alfredo Marcondes, Álvares Machado, Barretos, Batatais, Buritizal, Colina, Cristais Paulista, Guaraci, Jardinópolis, Mococa, Patrocínio Paulista, Pedregulho, Rifaina, Santo Antônio da Alegria e São Bento do Sapucaí). Nestes a prevalência da cisticercose bovina variou de 2,61\% a 7,94\% (Quadro 1). No entanto, quando se considera a maior prevalência aparente, 10 municípios (Mirante do Paranapanema, Terra Roxa, Gastão Vidgal, Nhandeara, Orlândia, Ribeirão Corrente, Santo Antônio do Jardim, Itirapina, Aramina e Mendonça) apresentaram índices entre 12,31\% a 31,58\% (Quadro 1).

Em relação aos núcleos regionais analisados, os três que obtiveram índices de prevalência mais elevados no Estado durante o período analisado, em ordem decrescente, foram: núcleo Central (9,38\%), Ribeirão Preto $(6,30 \%)$ e Presidente Prudente $(6,00 \%)$; já os núcleos de Sorocaba $(3,30 \%)$, Marília $(0,00 \%)$ e Araraquara $(0,00 \%)$ foram os três que apresentaram menores prevalências (Quadro 2). De acordo com os resultados descritos neste mesmo quadro, os núcleos de Araçatuba, Barretos, Bauru, Franca e Sorocaba apresentaram maior chance de se encontrar bovinos positivos para a cisticercose (Odds ratio $\mathrm{OR} \geq 1$ ).

A ocorrência da cisticercose variou entre os municípios dos núcleos regionais, e foi dependente da quantidade de animais abatidos proporcionalmente aos demais municípios do Estado. Levando em consideração os núcleos regionais do estado de São Paulo, os de Franca e Barretos, que juntos representaram $64,50 \%$ do total de animais abatidos neste estado, no referido período, apresentaram uma prevalência relativamente média de cisticercose $(4,5$ e 4,0\%, respectivamente), quando comparada com as demais (Quadro 2). Observando o número de casos de cisticercose bovina durante o período analisado, verifica-se que a maior prevalência desta enfermidade nos bovinos, aconteceu também nestes dois núcleos supracitados (Fig.1).

Quando se compara o total de casos de bovinos positi-

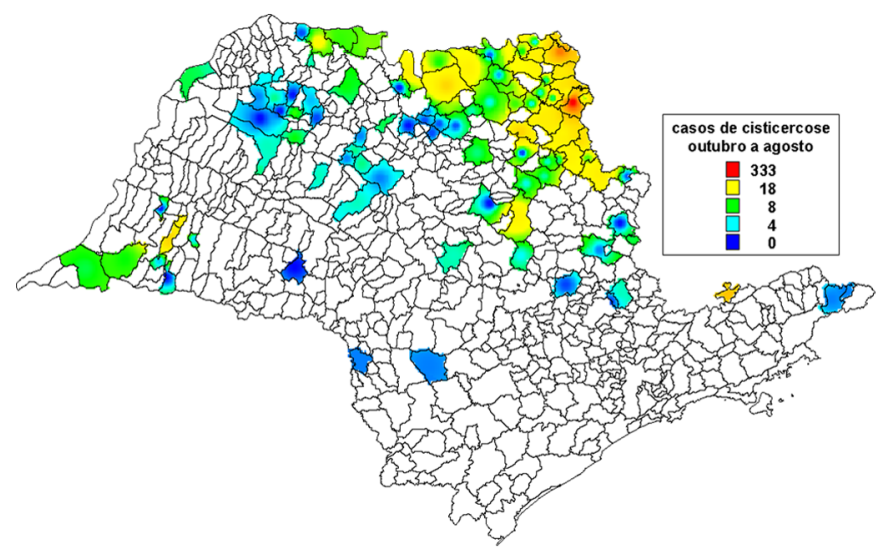

Fig.1. Distribuição espacial do número de casos de cisticercose bovina por município no estado de São Paulo no período de outubro de 2010 a agosto de 2011.
Quadro 1. Prevalência da cisticercose bovina aparente e em relação ao total de animais abatidos; Municípios que apresentaram maiores prevalências aparente de cisticercose bovina no período de outubro de 2010 a agosto de 2011

Município $\quad$ Prevalência (\%) $\quad$ Representatividade

$\begin{array}{lcc}\text { Municípios que tiveram maior quantidade de animais para abate } \\ \text { Colina } & 2,61 & 1,6 \\ \text { Guaraci } & 2,78 & 3,7 \\ \text { Rifaina } & 3,23 & 5,1 \\ \text { Barretos } & 4,09 & 4,8 \\ \text { Patrocínio Paulista } & 4,16 & 25,1 \\ \text { Batatais } & 4,67 & 2,3 \\ \text { Pedregulho } & 4,97 & 11,0 \\ \text { São Bento Do Sapucaí } & 5,07 & 5,1 \\ \text { Buritizal } & 6,72 & 1,6 \\ \text { Jardinópolis } & 6,73 & 2,9 \\ \text { Mococa } & 6,73 & 1,5 \\ \text { Cristais Paulista } & 6,81 & 1,6 \\ \text { Santo Antônio Da Alegria } & 6,83 & 2,3 \\ \text { Alfredo Marcondes } & 7,72 & 1,5 \\ \text { Álvares Machado } & 7,94 & 2,5\end{array}$

Municípios que apresentaram maiores prevalências aparente de cisticercose bovina

$\begin{array}{lll}\text { Mirante Do Paranapanema } & 12,31 & 0,19 \\ \text { Terra Roxa } & 12,50 & 0,12 \\ \text { Gastão Vidigal } & 12,50 & 0,23 \\ \text { Nhandeara } & 13,64 & 0,06 \\ \text { Orlândia } & 14,29 & 0,20 \\ \text { Santo Antônio Do Jardim } & 17,50 & 0,12 \\ \text { Itirapina } & 20,00 & 0,06 \\ \text { Ribeirão Corrente } & 22,22 & 0,05 \\ \text { Aramina } & 25,00 & 0,08 \\ \text { Mendonça } & 31,58 & 0,06\end{array}$

vos para cisticercose, no período em que os dados foram obtidos, com as variáveis: índice de desenvolvimento humano da educação por município (IDHE); área plantada de café; e total de cana-de-açúcar cultivada no estado, verifica-se que parte dos núcleos de Franca e Barretos foram os que tiveram menores índices de IDHE. Além disso, o núcleo de Franca é o que possuiu maior quantidade de área plantada de café, enquanto que o núcleo de Barretos conteve maior quantidade de cana cultivada, locais estes onde houve maior prevalência de bovinos positivos para cisticercose (Fig.2-4).

\section{DISCUSSÃO}

A prevalência de cisticercose bovina encontrada no presente estudo foi considerada menor que a relatada por Ungar \& Germano (1992) e baseada em observações no mesmo estado em 1986, quando os referidos autores diagnosticaram uma prevalência média de 5,5\%. De acordo com Guimarães-Peixoto et al. (2012), a prevalência da cisticercose bovina no Estado do Paraná esta diminuindo quando comparado aos dados de 1990. Segundo Falavigna-Guilherme et al. (2006) na década de 90 foram criados e implantados programas para a redução do complexo teníase-cisticercose em todo o estado do Paraná, além da implantação de abatedouros públicos municipais. Isto sugere, de acordo com os autores supracitados, que a implantação dessas medidas de controle e diagnóstico empregado no Estado favoreceram para a redução dos casos de cisticercose bovina. 
Quadro 2. Análise de associação entre os núcleos regionais do Estado de São Paulo, referente a prevalência de cisticercose bovina diagnosticada pelo Serviço de Inspeção Federal no período de outubro de 2010 a agosto de 2011

\begin{tabular}{|c|c|c|c|c|c|c|c|c|c|}
\hline \multirow{2}{*}{$\begin{array}{c}\text { Região } \\
\text { Administrativa }\end{array}$} & \multirow[t]{2}{*}{ Prevalência } & \multirow[t]{2}{*}{ Amplitude } & \multirow{2}{*}{$\begin{array}{l}\text { Número de } \\
\text { Municípios }\end{array}$} & \multirow{2}{*}{$\begin{array}{l}\text { Total de } \\
\text { bovinos } \\
\text { abatidos }\end{array}$} & \multirow{2}{*}{$\begin{array}{c}\text { Representatividade da região } \\
\text { em relação ao total de } \\
\text { bovinos abatidos }(\%)\end{array}$} & \multicolumn{2}{|c|}{ Cisticercose } & \multirow{2}{*}{$\begin{array}{l}\text { Odds ratio } \\
\qquad(\mathrm{OR})^{*}\end{array}$} & \multirow[t]{2}{*}{ IC 95\% } \\
\hline & & & & & & Negativo & Positivo & & \\
\hline Araçatuba & $3,5 \%$ & $0,0 \%$ a $12,5 \%$ & 8 & 1236 & $3,6 \%$ & 1193 & 43 & 1,40 & $1,03-1,91$ \\
\hline Araraquara & $0,0 \%$ & & 1 & 79 & $0,2 \%$ & 79 & 0 & & - \\
\hline Barretos & $4,0 \%$ & $0,0 \%$ a $12,5 \%$ & 11 & 4204 & $12,2 \%$ & 4036 & 168 & 1,21 & $1,03-1,43$ \\
\hline Bauru & $4,7 \%$ & $3,3 \%$ a $7,8 \%$ & 2 & 214 & $0,6 \%$ & 204 & 10 & 1,03 & $0,55-1,95$ \\
\hline Campinas & $5,9 \%$ & $0,0 \%$ a $20,0 \%$ & 9 & 957 & $2,8 \%$ & 901 & 56 & 0,81 & $0,62-1,07$ \\
\hline Central & $9,8 \%$ & & 1 & 328 & $1,0 \%$ & 296 & 32 & 0,47 & $0,32-0,68$ \\
\hline Franca & $4,5 \%$ & $1,5 \%$ a $25,0 \%$ & 21 & 18021 & $52,3 \%$ & 17209 & 812 & 1,07 & $0,98-1,17$ \\
\hline Marília & $0,0 \%$ & & 1 & 20 & $0,1 \%$ & 20 & 0 & & - \\
\hline Presidente Prudente & $6,0 \%$ & $0,0 \%$ a $12,3 \%$ & 9 & 2189 & $6,4 \%$ & 2058 & 131 & 0,79 & $0,66-0,95$ \\
\hline Ribeirão Preto & $6,3 \%$ & $0,0 \%$ a $20,0 \%$ & 11 & 3413 & $9,9 \%$ & 3197 & 216 & 0,75 & $0,65-0,87$ \\
\hline São José do Rio Preto & $4,9 \%$ & $0,0 \%$ a $31,6 \%$ & 19 & 1901 & $5,5 \%$ & 1808 & 93 & 0,98 & $0,79-1,22$ \\
\hline São José dos Campos & $5,1 \%$ & $4,4 \%$ a $5,1 \%$ & 3 & 1821 & $5,3 \%$ & 1728 & 93 & 0,94 & $0,76-1,16$ \\
\hline Sorocaba & $3,3 \%$ & & 1 & 60 & $0,2 \%$ & 58 & 2 & 1,46 & $0,36-6,00$ \\
\hline Total & $4,8 \%$ & & & 34443 & $100,0 \%$ & 32787 & 1656 & & \\
\hline
\end{tabular}

* Regiões administrativas com Odds ratio $(\mathrm{OR}) \geq 1$ apresentam maior chance de conter cisticercose bovina

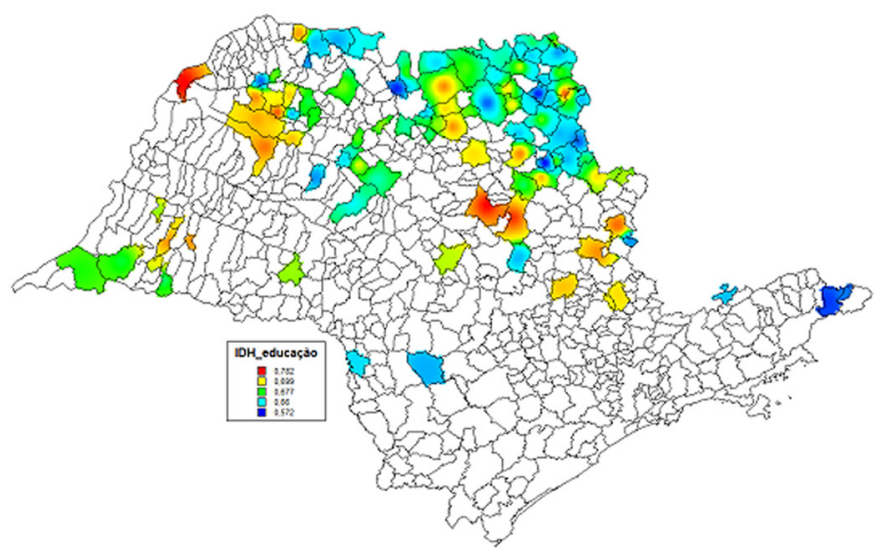

Fig.2. Distribuição espacial do índice de desenvolvimento humano da educação por município no estado de São Paulo no período de outubro de 2010 a agosto de 2011.

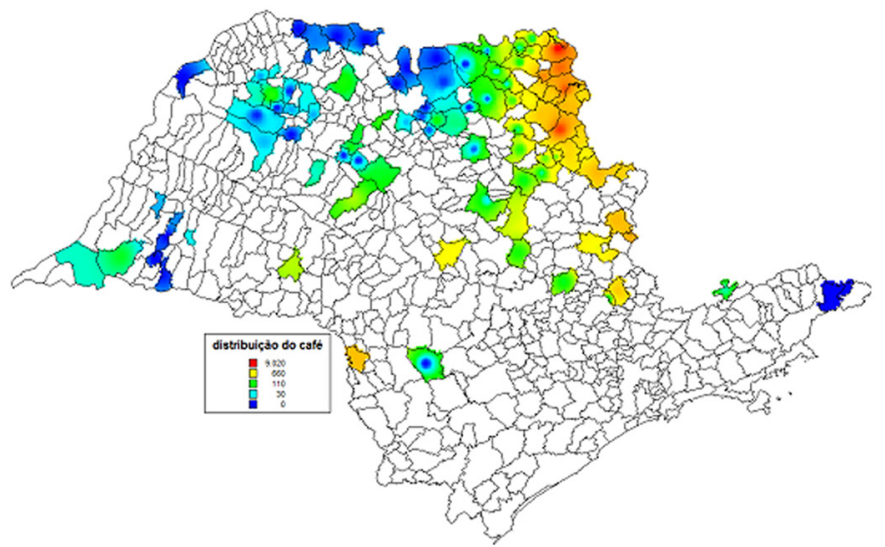

Fig.3. Distribuição espacial da área plantada de café por município no estado de São Paulo no período de outubro de 2010 a agosto de 2011.

0 fato de 11 municípios do estado de São Paulo terem sido classificados como áreas silenciosas ou sem registro de casos de bovinos com cisticercose, não representa necessariamente ausência desta enfermidade, especialmente quando existem evidências de sub-notificação, quer pela falta de estabelecimentos oficiais de inspeção (federal, estadual e

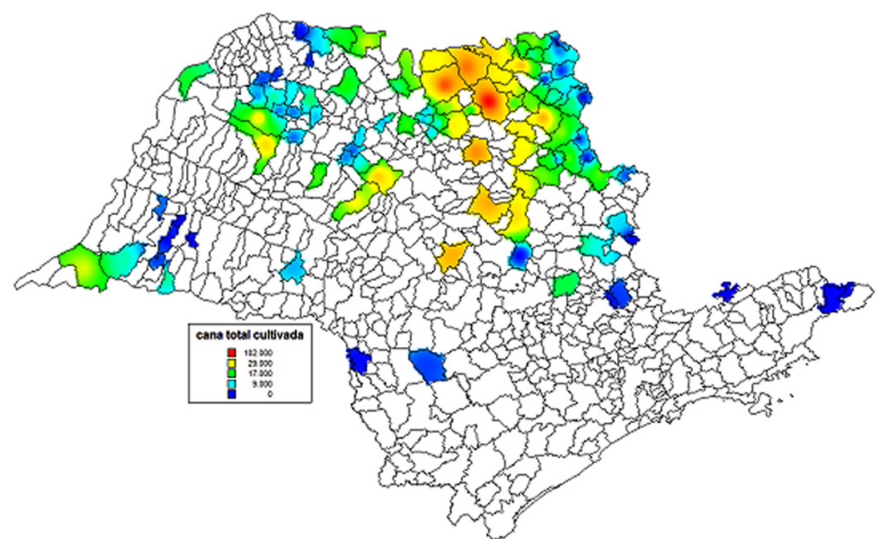

Fig.4. Distribuição espacial do total de cana-de-açúcar cultivada por município no estado de São Paulo no período de outubro de 2010 a agosto de 2011.

municipal), ou apenas pela presença de inspeção estadual ou municipal, ou, ainda, pela ocorrência do abate clandestino.

Souza et al. (2007) ressaltam que o maior número de casos de cisticercose bovina em São Paulo e Minas Gerais podem coincidir com uso intensivo de mão-de-obra temporária proveniente do meio rural. Os resultados encontrados no presente estudo reforçam tal inferência, uma vez que o maior número de casos bovinos com cisticercose foi encontrado nos núcleos de Franca e Barretos onde havia maior área plantada de café e também maior área de produção de cana-de-açúcar, respectivamente. Neste caso, muitas pessoas migram para o meio rural para realizarem a colheita das respectivas lavouras em locais onde a colheita mecânica não é possível, que geralmente são áreas vizinhas de pastagens contendo bovinos. Sendo assim, possíveis seres humanos portadores de teníase podem auxiliar na disseminação da cisticercose bovina, principalmente quando não existem banheiros químicos para estes trabalhadores temporários no meio rural. Apesar de não ser possível correlacionar, deve-se ressaltar ainda outro tipo de intensa migração de mão-de-obra temporária no meio rural, que é a realizada por pessoas que trabalham na construção e/ou reforma de rodovias no estado. 
Ungar \& Germano (1992), estudaram a prevalência da cisticercose bovina no estado de São Paulo também de acordo com a região administrativa, e encontraram como grandes áreas de risco as regiões administrativas de Ribeirão Preto, Bauru e Marília. Estes autores constataram ainda que, as áreas consideradas significativas para a presença da cisticercose bovina no estado em 1986, eram aquelas cujo à somatória do efetivo bovino dessas regiões representava 80,8\% do total do Estado. No presente estudo, foi possível verificar que o efetivo de gado dos núcleos de Franca e Barretos, correspondem a 3,6\% do efetivo total de bovinos do estado de São Paulo. Apesar da diferença entre os resultados deste estudo com os obtidos por Ungar \& Germano (1992), tal fato demonstra que, a presença da cisticercose bovina não necessariamente é dependente da presença do animal, mas sim da presença do ser humano infectado nas propriedades contendo bovinos, aliado às más condições higiênico-sanitárias, o baixo nível socioeconômico-cultural da região, ou ainda, da presença de mão-de-obra temporária no meio rural que também podem contribuir maciçamente para a persistente disseminação dessa enfermidade parasitária no campo. Nesse contexto, os matadouros desempenham papéis primordiais para a saúde pública, pois além de participam na prevenção da teníase humana, por meio da destinação apropriada de carcaças e órgãos bovinos cisticercóticos, atuam também como fonte de dados estatísticos e nosográficos (Ungar \& Germano 1992, Pereira et al. 2006), que são sinalizadores para a vigilância sanitária, pois permitem identificar as áreas de ocorrência da enfermidade para o direcionamento das ações de controle e de prevenção.

Os resultados encontrados neste estudo são importantes, de modo a serem tomadas medidas preventivas nas áreas de maior ocorrência da cisticercose bovina no estado de São Paulo. Medidas estas relacionadas à maior e melhor educação sociocultural das pessoas da região, e também dos trabalhadores rurais temporários que por ventura estejam nestes locais. A utilização de banheiros químicos para os trabalhadores temporários é uma medida que deve ser adotada. De qualquer maneira novos estudos em outras regiões do estado de São Paulo, que abrangem outros matadouros são necessários a fim de se avaliar outras possíveis interações socioeconômicas/cultural com esta enfermidade.

\section{CONCLUSÕES}

Com base nos resultados encontrados, pode-se concluir que a prevalência média de cisticercose bovina no estado de São Paulo durante o período analisado foi de 4,80\%, sendo que os núcleos de Franca e Barretos foram os que tiveram maior número de casos da enfermidade durante o período analisado.

O maior número de casos nestes núcleos coincidiu com o menor índice de desenvolvimento humano referente à educação, com a maior área de plantio de café (núcleo de Franca) e também como a maior produção de cana-de-açúcar cultivada (núcleo de Barretos) nestes locais, o que por sua vez pode indicar que a presença da mão-de-obra temporária no meio rural, aliado a aspectos socioeconômico/ cultural, podem estar contribuindo para a disseminação e estabelecimento da cisticercose bovina nestas áreas.

\section{REFERÊNCIAS}

Acha P.N. \& Szyfres B. 1986. Zoonosis y enfermedades transmisibles comunes ai hombre y a los aniniales. In: Anais Reunión Interamerinana el Organización Panamericana de la Salud, Washington, DC. Publicación Científica 503, WHO, Washington, DC.

Barbosa F.C., Cabral D.D. \& Sopelete M.C. 2003. Eficácia do sulfóxido de albendazol na cisticercose bovina. Hora Vet. 132:18-20.

Bavia M.E., Carneiro D.D.M.T., Cardim L.L., Silva M.M.N. \& Martins M.S. 2012. Estatística espacial de varredura na detecção de áreas de risco para a cisticercose bovina no estado da Bahia. Arq. Bras. Med. Vet. Zootec. 64:1200-1208.

Biondi G.F., Henrique C.H., Oliveira A.C. \& Castro A.P. 2000. Avaliação da eficiência da prova de evaginação em metacestódeos de Taenia saginata. Revta Educ. Cont. CRMV-SP 3:55-61.

Brasil 1971. Padronização de Técnicas, Instalações e Equipamentos. Vol.1. Bovinos. Serviço de Inspeção de produtos de Origem Animal (DIPOA), Ministério da Agricultura Pecuária e Abastecimento (MAPA), Brasília, DF, p.78-95.

Brasil 1980. Regulamento de Inspeção Industrial e Sanitária de Produtos de Origem Animal. DIPOA, Ministério da Agricultura, Brasília.

Brasil 1988. Trabalhos científicos sobre cisticercose. Circular no. 54 de 19 maio 1988. Divisão de Inspeção de Carnes (DIPOA), Ministério da Agricultura, Brasília, DF.

Brasil 1997. Relação de abate de doença por procedência e município. Serviço de Inspeção Federal (SIF), Ministério da Agricultura. Disponível em $<$ http://extranet. agricultura.gov.br> Acesso em 15 set. 2006.

Costa R.F.R., Santos I.F., Santana A.P., Tortelly R., Nascimento E.R., Fukuda R.T., Carvalho E.C.Q. \& Menezes R.C. 2012. Caracterização das lesões por Cysticercus bovis, na inspeção post mortem de bovinos, pelos exames macroscópico, histopatológico e pela reação em cadeia da polimerase (PCR). Pesq. Vet. Bras. 32:477-484.

Falavigna-Guilherme A.L., Silva K., Araújo S.M., Tobias M.L. \& Falavigna D.L.M. 2006. Cisticercose em animais abatidos em Sabáudia, Estado do Paraná. Comunicação. Arq. Bras. Med. Vet. Zootec. 58:950-951.

Guimarães-Peixoto R.P.M., Souza V.K., Pinto P.S.A. \& Santos T.O. 2012. Distribuição e identificação das regiões de risco para a cisticercose bovina no Estado do Paraná. Pesq. Vet. Bras. 32:975-979.

Lopes W.D.Z., Santos T.R., Soares V.E., Nunes J.L.N., Mendonça R.P., Lima R.C. A., Sakamoto C.A.M., Costa G.H.N., Thomaz-Soccol V.T., Oliveira G.P. \& Costa A.J. 2011. Preferential infection sites of Cysticercus bovis in cattle experimentally infected with Taenia saginata eggs. Res. Vet. Sci. 90:84-88.

Lopes W.D.Z., Cruz B.C., Soares V.E., Nunes J.L.N., Teixeira W.F., Maciel W., Buzulini C., Pereira J.C., Felippelli G., Soccol V.T., Oliveira G.P. \& Costa A.J. 2014. Historic of therapeutic efficacy of albendazol sulphoxide administered in different routes, dosages and treatment schemes, against Taenia saginata cysticercus in cattle experimentally infected. Exp. Parasitol. 137:14-20.

Pereira M.A.V.C., Schwanz V.S. \& Barbosa C.G. 2006. Prevalência da Cisticercose em Carcaças de Bovinos Abatidos Matadouros-Frigoríficos do Estado do Rio de Janeiro, Submetidos ao controle do Serviço de Inspeção Federal (SIF-RJ), no período de 1997 a 2003. Arqs Inst. Biológico, São Paulo, 73:83-87.

Schantz P.M., Cruz M., Sarti È. \& Pawlowski Z.S. 1994. La erradicabilidad potencial de la teniasis y la cisticercosis. Boln Of. San. Panam. 5(16):465-458.

Souza W.K., Pessôa-Silva M.C., Minozzo J.C. \& Thomaz-Soccol V. 2007. Prevalência da cisticercose bovina no estado do Paraná, sul do Brasil: avaliação de 26.465 bovinos inspecionados no SIF 1710. Semina, Ciênc. Agrárias 28:675-684.

Ungar M.L. \& Germano P.M.L. 1992. Prevalência da cisticercose bovina no Estado de São Paulo, Brasil. Revta Saúde Publ. 26:167-172.

Wanzala W., Onyargo J.A., Kang E.K., Ochanda H. \& Harison L.J.S. 2002. Serodiagnosis of bovine cysticercosis by detecting live Taenia saginata cysts using a monoclonal antibody-based antigen-ELISA. J. South Afr. Vet. Assoc. 73:201-206. 